\title{
SISTEMAS DE REPRESENTAÇÕES SOCIAIS: contribuições para a pesquisa em educação
}

\author{
Caio Teixeira Brandão \\ Andréa Silva Benevides \\ Pedro Humberto Faria Campos
}

\section{Resumo}

Este artigo apresenta algumas contribuições da noção de sistemas de representações sociais (SRS) para a pesquisa em Educação, a partir de duas pesquisas com base na Abordagem Estrutural, que entende a representação social como um sistema de crenças que orienta e justifica as práticas de um determinado grupo. Em uma pesquisa no campo da Educação Profissional, por meio da Técnica de Associação Livre de Palavras (TALP) e da Análise Prototípica, foram identificados indícios da relação entre ensino técnico e capacitação profissional para alunos de cursos técnicos de nível médio de um Instituto Federal de Educação, que se estabelece a partir de uma noção polissêmica de oportunidade. Numa pesquisa-ação em Educação para a Saúde, realizada com funcionários de uma rede de supermercados, o uso de grupo focal permitiu identificar a relação entre saúde e atividade física e intervir para aumentar a adesão dos participantes à prática de exercício físico. A perspectiva sistêmica se contrapõe ao estudo de representações isoladas e oferece uma alternativa para o estudo das representações sociais, favorecendo um olhar psicossocial na intervenção e transformação das práticas educativas.

Palavras-chave: sistemas de representações sociais; abordagem estrutural; pesquisa em educação.

SOCIAL REPRESENTATIONS SYSTEMS: contributions to educational research

\begin{abstract}
This paper presents some contributions of social representation systems (SRS) to research in Education, using two researches based on the Structural Approach, which establishes socials representations as a belief system that guides and justifies the practices of a group. In a research in the field of Professional Education, through the Free Word Association Technique and the Prototypical Analysis, evidence was found of the relationship between technical education and professional training for students of high school courses of a Federal Institute of Education, based on a controversial notion of opportunity. In an action research in Health Education, conducted with employees of a supermarket chain, the use of a focus group made it possible to identify the relationship between health and physical activity and intervene to increase participants' adherence to the practice of physical exercise. The systemic perspective opposes the study of isolated representations and offers an alternative for the study of social representations, favoring a psychosocial regard in intervention and transformation of educational practices.
\end{abstract}

Keywords: social representation systems; structural approach; educational research. 


\title{
SISTEMAS DE REPRESENTACIÓN SOCIAL:
}

\author{
contribuciones en la investigación en educación
}

\section{Resumen}

Este artículo presenta algunas contribuciones de la noción de sistemas de representación social (SRS) a la investigación en Educación, a partir de dos investigaciones basadas en el Enfoque Estructural, que entiende la representación social como un sistema de creencias que guía y justifica las prácticas de un grupo determinado. En una investigación realizada en el ámbito de la educación profesional, mediante la técnica de asociación de palabras libres (TALP) y el análisis prototípico, se identificaron pruebas de la relación entre la educación técnica y la formación profesional de los estudiantes de los cursos de la escuela secundaria técnica de un Instituto Federal de Educación, que se establece a partir de una controvertida noción de oportunidad. En una investigación de acción en materia de educación sanitaria, realizada con empleados de una cadena de supermercados, el uso de un grupo de discusión permitió identificar la relación entre la salud y la actividad física e intervenir para aumentar la adhesión de los participantes a la práctica del ejercicio físico. La perspectiva sistémica se opone al estudio de las representaciones aisladas y ofrece una alternativa para el estudio de las representaciones sociales, favoreciendo una mirada psicosocial en la intervención y transformación de las prácticas educativas.

Palabras clave: sistemas de representaciones sociales; enfoque estructural; investigación en educación.

\section{DO CONCEITO AO FENÔMENO DE REPRESENTAÇÕES SOCIAIS}

A Teoria das Representações Sociais (TRS), criada por Moscovici (2012) no início da década de 1960, possui hoje uma rede mundial de pesquisadores e eventos, a maioria distribuída nas áreas de educação e saúde. Para Sousa (2003), o sucesso da teoria deve-se a uma visão de sujeito historicamente situado, interface entre o micro e o macro, em substituição às teorias psicológicas eminentemente individualizantes. A representação reflete as condições sociais e históricas nas quais emerge, nela são mobilizados elementos do contexto e da cultura para a construção de um sistema de interpretação da realidade que, comum aos membros do grupo, orienta a conduta e a comunicação, mediando a relação entre o sujeito e o objeto representado (JODELET, 1984).

A passagem da noção para o conceito e a construção de uma teoria própria das representações sociais é relatada por Jodelet (1984), que agrupa sob o nome de representações sociais uma diversidade de fenômenos, indicando como característica principal o fato de serem uma modalidade de conhecimento social de caráter prático, interface entre o psicológico e o social, que contribui para a construção de uma realidade consensual.

No âmbito da TRS, os pesquisadores comumente abordam os objetos de representação de forma isolada, no intuito de investigar o conhecimento produzido pelos grupos em relação a determinado objeto da realidade social. Os estudos são frequentemente intitulados: "a representação social de determinado objeto para esse ou aquele grupo" e embora sejam identificadas como uma forma de pensamento social, Garnier (2015) aponta as contradições teórico-epistemológicas das pesquisas, ao considerarem indivíduos e objetos isolados como unidades de análise. Flament e Rouquette (2003) afirmam que o estudo de representações isoladas é uma escolha empírica que responde à necessidade de simplificar os modelos e os processos implicados no fenômeno, tendo em vista que na realidade social os objetos não possuem fronteiras bem definidas.

Em um levantamento sobre o uso da noção de sistema de representações sociais na literatura nacional e internacional, Félix, Andrade e Santos et al. (2016) identificaram uma quantidade reduzida de trabalhos que se referem especificamente ao tema, embora tenham observado um crescimento no uso do termo sistema nos últimos anos. As autoras identificaram uma 
variedade de conceitos, tais como, conjuntos, famílias ou redes de representação, que se referem à organização das representações sociais a partir das relações estabelecidas entre os elementos. No entanto, prevalece o emprego da noção de sistemas de representações sociais como as "[...] articulações entre representações e/ou objetos sociais, podendo ser identificado a partir do compartilhamento de elementos" (FÉLIX, ANDRADE, SANTOS et al., 2016, p. 208).

Não obstante os dados da pesquisa reflitam, em parte, as limitações das bases de dados e dos repositórios utilizados, capazes de efetuar a busca apenas por palavras-chave, eles também sinalizam a ausência constatada por Wachelke (2016) em artigos científicos, que, embora relatem a investigação da relação entre elementos ou mesmo entre representações sociais, não fazem alusão ao conceito de sistemas de representações sociais. Estudos tendo como referência a Abordagem Estrutural (PIANELLI et al., 2010; CAMARGO, WACHELKE, 2010; WACHELKE, CONTARELLO, 2011) têm contribuído para a investigação empírica das relações entre os objetos sociais, a partir da concepção da representação social enquanto duplo sistema, central e periférico, e da distribuição dos elementos cognitivos na estrutura da representação.

Esse artigo tem por objetivo apresentar a noção de sistemas de representações sociais e suas possíveis contribuições para a Educação, a partir de duas pesquisas, uma pesquisa exploratória sobre educação profissional e uma pesquisa-ação no âmbito da educação em saúde. A noção de sistemas de representações sociais pode auxiliar na compreensão e construção de modelos interpretativos dos fenômenos e das práticas, aprofundando o olhar psicossocial na pesquisa em Educação (SOUSA, NOVAES, VILLAS BÔAS, 2012), tão necessário nesse campo marcado por uma trajetória histórica de lutas e conflitos.

\section{O USO DA NOÇÃO DE SISTEMA NA TRS}

Moscovici (2012) apresentou a representação social inicialmente como um conjunto organizado de crenças descritivas e prescritivas, uma totalidade distinta de outras formas de pensamento social, também chamada de conhecimento cotidiano ou senso comum, que permite a interação e comunicação entre os membros de um grupo em relação a determinado objeto da realidade social.

$\mathrm{Na}$ gênese da representação social, dois processos fundamentais, ancoragem e objetivação, seriam responsáveis pela dialética da transformação do conhecimento em representação e a consequente mudança da realidade a partir da representação (JODELET, 1984). Esses dois mecanismos atuam de forma complementar: a objetivação condensa os elementos da representação em uma imagem ou núcleo figurativo, permitindo a sua concretização e naturalização, enquanto a ancoragem permite a categorização e integração dos objetos não-familiares ao conhecimento previamente produzido pelo grupo.

Embora Moscovici (2012), em seu estudo pioneiro “A psicanálise: sua imagem e seu público" tenha se referido ao papel da ancoragem e da objetivação na gênese de uma representação social, mostrando a apropriação da Psicanálise pela sociedade francesa da década de 1960, é possível notar ao longo do tempo uma proliferação de estudos que se ocupam apenas da investigação do conteúdo da representação no presente, negligenciando a dimensão histórica do fenômeno. Mas o estudo da ancoragem permitiria identificar a rede de significados em torno do objeto, dando ênfase na inserção do fenômeno de representação em um conjunto de expressões socioculturais e históricas, enquanto a objetivação permitiria a delimitação das fronteiras de uma representação social, seja por meio da hipótese do núcleo figurativo ou do núcleo central, e sua materialização na realidade, subordinada aos valores do grupo. 
A Abordagem Estrutural, ou Teoria do Núcleo Central, desenvolvida a partir dos trabalhos de Jean-Claude Abric (2003) e Claude Flament (1994), pesquisadores da Escola de Aix-enProvence, estabelece a representação social enquanto sistema sociocognitivo que possui uma estrutura hierarquizada, formada por um duplo sistema: núcleo central e sistema periférico, cada um com elementos e funções distintas. Enquanto o núcleo central seria o responsável pela caracterização do objeto, o sistema periférico constituiria o aspecto operacional da representação, permitindo sua adaptação aos diferentes contextos sociais.

\section{A representação social como sistema estruturado}

A evolução do núcleo central enquanto estrutura estática para a concepção de sistema central está presente no estudo de Rateau (1995) sobre a representação do grupo ideal, talvez o objeto mais pesquisado pelos estudiosos franceses da abordagem estrutural. A partir dos resultados de pesquisas anteriores e do uso do teste de questionamento (mise-en-cause), que permite a investigação dos elementos centrais da representação, considerados indispensáveis para a identificação do objeto, o autor postulou que existe uma assimetria entre os elementos do núcleo central da representação social, que seria composto por dois tipos de elementos: os prioritários, responsáveis pela significação do objeto, e os adjuntos, de caráter mais normativo, responsáveis pela particularização das representações (RATEAU, LO MONACO, 2016). Ambos os elementos, prioritários e adjuntos, fariam parte do sistema central, porém, somente os prioritários seriam incondicionais, resistindo aos ataques do teste de centralidade.

Rateau (1995) também constrói a hipótese de que alguns objetos sociais estariam organizados em uma rede de representações, observada a partir da relação que se estabelece entre diferentes representações num mesmo grupo, organizadas em torno de elementos comuns (ou elementos principais) que fazem a ligação entre as representações. Dessa forma, a significação das representações em rede estaria condicionada aos elementos compartilhados.

$\mathrm{Na}$ estrutura dupla da representação social, a periferia também pode ser entendida como um sistema (FLAMENT, 1994), com algumas funções específicas: ela permite aos indivíduos reconhecer a conduta desejável em determinadas situações; possibilita a personalização ou modelização individual da representação dentro de um grupo, desde que não contradiga os elementos centrais; e protege o núcleo central diante das mudanças, servindo como uma espécie de para-choque, absorvendo o impacto e adaptando-se à situação. Segundo o autor, é na dialética entre os elementos centrais e periféricos que se dá o funcionamento da representação, e é a partir do sistema periférico que se inicia a transformação de uma representação social.

\section{A relação entre representações e práticas sociais na perspectiva sistêmica}

A relação entre representações e práticas sociais tem sido objeto de estudo da abordagem estrutural, devido ao potencial das práticas na transformação da representação social. O primeiro modelo de investigação da relação entre representações sociais e práticas enquanto sistema teria sido proposto por Codol (1974), num modelo denominado Representação Global da Situação, no qual o autor propõe que a ação dos grupos em situações reais se dá como consequência da relação entre a representação que o grupo possui de si mesmo, a representação do outro e a representação da tarefa. Para entender a dinâmica da situação num contexto de relações intergrupais, seria necessário conhecer o conjunto de representações e de práticas implicadas.

$\mathrm{Na}$ relação que se estabelece entre a representação social e as práticas desenvolvidas pelo grupo, podem constituir-se verdadeiros sistemas de interpretação da realidade e, de acordo com o 
contexto, diferentes elementos da representação são ativados, prescrevendo práticas que permitem a manutenção ou mesmo a transformação da representação.

O modelo de Codol influenciou os estudos acerca do fenômeno de exclusão-inclusão (ABRIC, CAMPOS, 1996; CAMPOS, 2003), nos quais se faz necessário entender a dinâmica entre os diferentes grupos envolvidos para a compreensão do fenômeno, pois a exclusão de um grupo só pode ocorrer na perspectiva da existência de outros grupos "incluídos". A partir da vivência de educadores e "meninos-de-rua", Campos (2003) desenvolveu um modelo de sistema representacional voltado para o estudo da relação entre grupos e práticas em contextos de exclusão, considerando fatores como a percepção de autonomia do grupo sobre a sua prática, o contexto institucional, a representação que o grupo possui de si, a representação do grupo excluído e a carga afetiva relacionada à atividade e memória coletiva.

\section{A relação entre representações sociais como uma forma de sistema}

Num estudo sobre a relação entre a Teoria das Representações Sociais e a Teoria da Identidade Social, Breakwell (1993) afirma que as representações sociais são dinâmicas e estariam sujeitas à mudança, como resultado de uma articulação em rede e da relação entre os objetos na realidade social, o que para a autora estaria implícito no conceito de ancoragem. Ela ressalta que a forma pela qual se delimitam as fronteiras de uma representação é uma decisão arbitrária do pesquisador, e propõe a articulação entre a TRS e a Teoria da Identidade Social, como formas complementares de estudo dos processos grupais. Enquanto a TRS aborda o fenômeno do ponto de vista da construção coletiva de conhecimento, do grupo para o indivíduo, a Teoria da Identidade Social refere-se à construção da identidade dos atores sociais por meio da diferenciação com os outros e da atribuição social, num continuum entre a identidade individual e a identidade social. Segundo a autora, no estudo das representações sociais, devem-se considerar as relações de poder intra e intergrupos, pois esses aspectos seriam determinantes na adesão dos indivíduos às representações e no sentimento de pertencimento aos grupos.

O uso do termo sistema representacional para designar um conjunto de representações ou a relação entre duas ou mais representações teria sido inicialmente empregado por Garnier (1999), em uma pesquisa sobre corpo, saúde e ambiente realizada com crianças de berçário e em idade escolar, na qual a autora teria identificado o desenvolvimento de representações e sua estruturação em um sistema referente às relações humanas. A perspectiva de sistemas representacionais permitiria contextualizar as representações sociais e a relações entre os grupos.

A definição de sistema de representações sociais é assim apresentada por Silva, Trindade e Júnior (2012, p. 441):

[...] um sistema de representações pode ser entendido como um conhecimento socialmente partilhado formado por um conjunto de objetos representacionais, que, por sua vez, são constituídos por um conjunto de elementos representacionais. Todos os objetos e os elementos representacionais presentes em um sistema representacional estão interrelacionados de tal forma a dar coerência e sentido a esse sistema representacional, como uma rede de significações e símbolos.

Essa definição é resultado de uma pesquisa sobre recasamento e novas configurações familiares, na qual as autoras identificaram que as representações dos participantes sobre o papel dos membros da família, inclusive do novo cônjuge e dos filhos do primeiro casamento, estavam diretamente relacionadas à representação de família dos participantes. 
Flament e Rouquette (2003) classificaram as possíveis relações entre representações sociais em dois tipos: conjunção, nas quais ocorre uma intersecção entre os núcleos centrais de duas representações ou mais, e disjunção, quando não há intersecção entre os núcleos. Já Abric (2003) postula que as relações entre representações sociais podem ser de encaixe (do francês emboîtement), constituindo um sistema hierarquizado, onde há uma relação de subordinação entre representações; e de reciprocidade, no qual os objetos sociais estão presentes como elementos do sistema central de ambas as representações sociais, sendo essenciais para sua significação. Posteriormente, foi acrescentada a esse grupo a relação de antinomia, quando as representações estabelecem uma relação de oposição entre si.

Os estudos de Flament e Rouquette (2003) sobre a arquitetura do pensamento social fornecem uma possibilidade para identificar a natureza das relações entre as representações sociais. Segundo o modelo proposto pelos autores, as formações simbólicas estariam organizadas hierarquicamente, sendo as mais amplas e abstratas responsáveis por orientar a construção de sentido das mais particulares, situando-se os construtos ideológicos no nível superior e as opiniões no nível inferior. A partir desse modelo, as relações entre representações sociais dar-se-iam no mesmo nível de hierarquia, e os sistemas de representação construir-se-iam em torno das representações subordinadas a um mesmo tema imediatamente superior na hierarquia do pensamento social.

A hipótese da ancoragem de novos objetos de representação em representações já existentes foi levantada por Pianelli et al. (2010) em uma pesquisa sobre a implementação e a aceitabilidade do sistema de limitação de velocidade LAVIA $^{1}$ entre os condutores franceses de automóveis. Dentre as possíveis relações entre representações sociais, a relação de encaixe seria uma etapa necessária da gênese de novas representações, pois seria a forma como as representações em formação se estruturariam.

[...] le processus d'ancrage d'une nouvelle représentation dans le système de pensée peut être considéré comme l'emboîtement de cette représentation dans une ou plusieurs représentations préexistantes. Les représentations «emboîtantes» devraient ainsi déterminer le contenu et la structure de toute nouvelle représentation «emboitée». Les représentations sociales préexistantes auraient ainsi un rôle crucial à cette étape de la genèse représentationnelle (PIANELLI et al., 2010, p. 245)2.

Dessa forma, a consolidação de novas representações se daria a partir das redes ou sistemas de representações sociais, tendo em vista que as representações "encaixadas" se estruturam a partir das representações nas quais estariam ancoradas. Os autores demonstraram, a partir da pesquisa empírica, que a aceitabilidade do sistema LAVIA dependia da atitude e da relação estabelecida pelos grupos de condutores franceses entre velocidade e limitação de velocidade, objetos diretamente relacionados, mas que estabeleciam entre si uma relação de antinomia.

Pianelli e Saad (2016) relatam como principal obstáculo à pesquisa de representações em formação, ou em fase de estruturação, a dificuldade para se identificar os objetos de representação que possivelmente compõem o conjunto ou rede no qual a nova representação estaria ancorada, sendo necessário um conhecimento prévio do campo do objeto, um dos aspectos da representação

\footnotetext{
${ }^{1}$ Do francês: Limiteur s'Adaptant à la vitesse Autorisée (LAVIA), que poderia ser traduzido como Limitador que se adapta à velocidade autorizada.

$2 \mathrm{O}$ processo de ancoragem de uma nova representação no sistema de pensamento pode ser considerado como o encaixe dessa representação em um ou mais representações preexistentes. As representações "estruturantes" determinam o conteúdo e a estrutura da nova representação "encaixada". As representações sociais preexistentes têm assim um papel crucial nessa etapa da gênese representacional. (PIANELLI et al., 2010, p. 245, tradução nossa).
} 
social, conforme estabelecido por Moscovici (2012), ao lado da informação e da atitude do grupo em relação ao objeto.

\section{Sistemas de representações sociais no campo da educação}

As representações sociais construídas no campo da educação referem-se a objetos de estudo fortemente marcados pelo contexto histórico e cultural, "carregados" afetiva e simbolicamente; dada essa natureza, são muito difíceis de ser analisados com base no estudo de uma representação isolada. Um conjunto de estudos recentes sobre a representação do futuro em diferentes estratos do campo educacional, organizado por Sousa et al. (2018), ilustra essa perspectiva. Apesar de não aplicar a noção de "sistema de representações" os estudos mostram uma interdependência da representação social do futuro em relação a outros elementos representacionais presentes em cada contexto, sejam a escola e a carreira docente; a juventude e as transformações da sociedade; ou a formação universitária e sua dimensão afetiva. Essas pesquisas atuais, no campo da educação escolar, mostram como, para se compreender o conteúdo e implicações de uma representação, faz-se necessário conhecer outros elementos representacionais que são percebidos como importantes para os sujeitos em cada contexto específico.

Desde o estudo clássico de Michel Gilly (1980) sobre a relação professor-aluno, reconhecese o papel das representações sociais nas relações intersubjetivas. No contexto da aprendizagem escolar, estão em jogo representações recíprocas, as representações do professor sobre si mesmo e do "aluno representado"; e essas estão em contraste com o aluno "real" e suas representações sobre o papel do professor e da escola. Essas representações sofrem influência das condições institucionais, e são constantemente atualizadas a partir da experiência dos sujeitos, pois, na construção da representação de si mesmo, a existência do outro é uma condição essencial, não apenas como destinatário do discurso, mas como sujeito-objeto de um processo de categorização e particularização.

Em um estudo sobre os educadores de "meninos de rua", Campos (2003) percebeu que as práticas desenvolvidas pelos agentes poderiam ser compreendidas a partir de um sistema representacional, no qual interagem os elementos centrais das representações do papel do educador e dos "meninos de rua" e as concepções institucionais. Nesse contexto, a representação dos meninos se destacava como princípio organizador do sistema, em torno do qual se articulavam as demais representações, pois as práticas relacionadas à representação dos "meninos de rua" dividiam-se entre aquelas direcionadas à família e aquelas relacionadas ao combate da exclusão e da miséria, enquanto as práticas relacionadas ao papel do educador estavam associadas aos elementos "agente de transformação", defesa de direitos, aconselhamento e engajamento das famílias e dos meninos.

A partir do questionamento de professores do estado de Pernambuco, Bona, Espíndola e Maia (2017) identificaram indícios de uma possível relação de encaixe entre autonomia e autonomia docente. Embora não tenham realizado um estudo estrutural aprofundado, as autoras construíram a hipótese de que a presença de elementos possivelmente centrais, comuns a ambas as representações, sugere uma dependência entre os objetos. A autonomia docente estabeleceria uma relação hierárquica, porém dialética, com a representação que os professores possuem de autonomia, enquanto fim pedagógico que orienta as suas práticas.

No estudo de Perez (2017) sobre a relação entre trabalho docente, papel institucional e as práticas desenvolvidas por professores dos anos iniciais da educação básica, a autora propõe que a atividade docente se inscreve num sistema de representações do qual fazem parte a percepção dos professores sobre as condições objetivas da escola; a representação que o professor possui de si 
mesmo; o papel institucional, conforme preconizado pela escola; e as práticas docentes. A autora submeteu a um teste de centralidade os elementos considerados centrais na representação de trabalho docente: amor, dedicação e compromisso (identificados em pesquisas anteriores). $\mathrm{O}$ trabalho docente apresentaria um caráter ambivalente, devido ao conflito existente entre a função do professor e as expectativas da escola, assumindo os elementos centrais da representação a função de salvaguardar uma identidade positiva do docente, frente à desvalorização que a profissão tem sofrido nos últimos anos.

Os resultados da pesquisa apontam que as práticas valorizadas pelos professores pouco têm em comum com aquilo que consideram ser o papel institucional, as atividades que a escola espera que desenvolvam. As práticas valorizadas pelo professor estariam relacionadas à operacionalização das aulas, enquanto aquelas valorizadas pela escola seriam relacionadas a questões burocráticas, como o preenchimento de diários de classe e cadernos de frequência. O ponto de confluência entre o professor e a escola estaria nas práticas relacionadas à manutenção da disciplina e do bom comportamento dos alunos.

\section{REPRESENTAÇÕES SOCIAIS NO CAMPO DA EDUCAÇÃO PROFISSIONAL}

Em uma pesquisa com o objetivo de investigar a possível relação entre Ensino Técnico e Capacitação Profissional para alunos de cursos técnicos (BRANDÃO, 2016), a partir da técnica de associação livre de palavras (TALP), foram identificados os principais elementos associados aos termos ensino técnico e capacitação profissional, e foi traçado um perfil socioeconômico dos alunos, utilizando critérios como renda per capita familiar e grau de escolaridade.

Participaram da pesquisa 75 alunos de cursos técnicos de nível médio e nível superior de um programa de concessão de auxílio financeiro de uma instituição da Rede Federal de Educação Profissional e Tecnológica, que responderam a um questionário com dois termos indutores para a associação verbal e perguntas de múltipla escolha para traçar um perfil socioeconômico. A tarefa de associação consistia em responder a pergunta: "[...] quais as cinco palavras ou expressões que lhe vêm à mente quando você pensa em ensino técnico?" e, posteriormente, o mesmo procedimento foi repetido com o termo capacitação profissional. Como parte da tarefa de associação, os participantes também deveriam assinalar a palavra considerada mais importante e justificar sua escolha.

A pesquisa foi realizada num Instituto Federal de Educação, Ciência e Tecnologia (IF), estabelecimentos de educação profissional e tecnológica criados no ano de 2008 por meio da Lei n. 10.892/2008 (BRASIL, 2008). Na realidade socialmente construída, o novo nem sempre é estranho, mas apenas um fenômeno para o qual ainda não existem referências no grupo; e a implantação do Instituto Federal, que iniciou suas atividades escolares em 2010, aliada a uma proposta de integração do ensino técnico ao ensino médio, afigurou-se como uma novidade para a população local.

Os resultados da pesquisa permitem realizar inferências a respeito da possível relação entre ensino técnico e capacitação profissional para os alunos da referida instituição, a partir dos dados da análise prototípica e do quadro de quatro casas construído com o auxílio do software Evoc Simi $2016^{3}$. A análise prototípica consiste na distribuição das palavras associadas pelos participantes em um gráfico composto por quatro quadrantes, por meio dos critérios de frequência e ordem média

\footnotetext{
${ }^{3}$ Programa computacional desenvolvido pela Universidade Estácio de Sá em parceria com o CNPq e o INTO que auxilia na análise de evocações (análise prototípica) coletadas por meio de técnica de associação livre de palavras e análise de similitude.
} 
de evocação (OME). Essa técnica configura uma etapa inicial da pesquisa estrutural que tem por objetivo os possíveis elementos do núcleo central da representação que se destacam pela alta frequência e são prontamente evocados.

\section{A relação entre Ensino Técnico e Capacitação Profissional}

$\mathrm{Na}$ distribuição das palavras associadas pelos participantes ao termo indutor ensino técnico (Figura 1) foram indicados como possíveis elementos centrais da representação capacitação, oportunidade e formação. O quadrante de quatro casas é fortemente marcado por elementos referentes à formação profissional, como oportunidade de ingresso no mercado de trabalho, dentre eles a ideia de capacitação como experiência adquirida ao longo da formação, elemento presente nas justificativas dos alunos.

Figura 1. Quadrante das evocações ao termo ensino técnico

\begin{tabular}{|c|c|c|c|c|c|}
\hline $\begin{array}{l}\text { OME } £ 2.74 \\
f^{3} 16 \\
\text { Núcleo Central }\end{array}$ & & ome & $\begin{array}{l}\text { OME }>2.74 \\
\mathrm{f}^{3} 16 \\
\text { Periferia Próxima }\end{array}$ & f & ome \\
\hline capacitação & 24 & 2.21 & conhecimento & 17 & 3.71 \\
\hline oportunidade & 24 & 2.71 & mercado-de-trabalho & 16 & 3.0 \\
\hline formação & 16 & 2.44 & & & \\
\hline
\end{tabular}

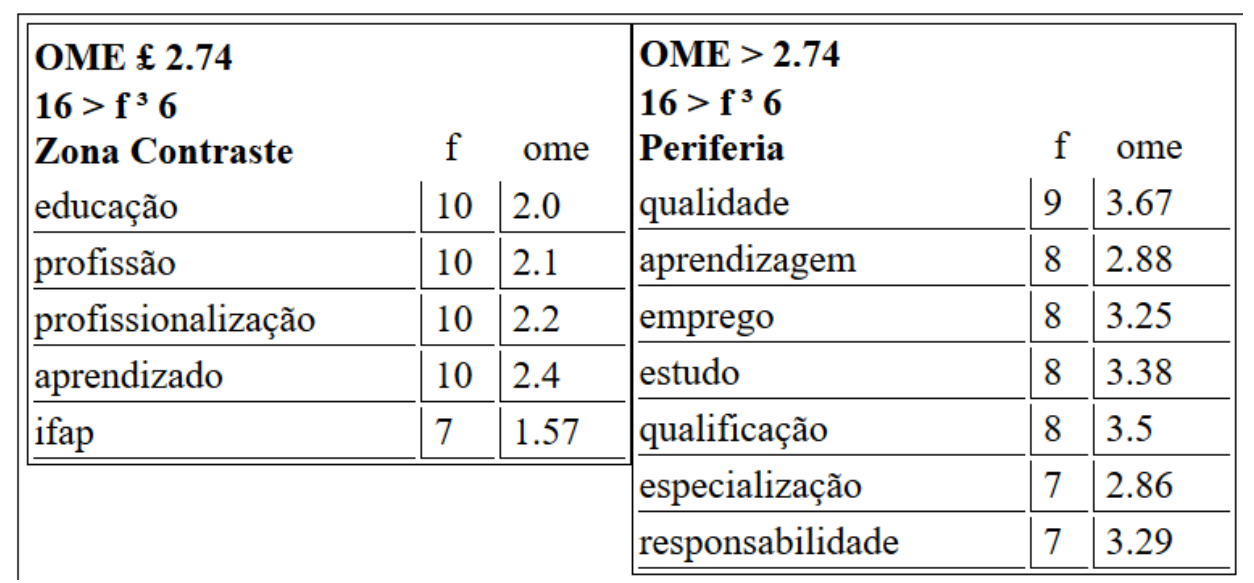

Fonte: Brandão, 2016

No caso das palavras associadas ao termo indutor capacitação profissional (Figura 2), destacam-se como possíveis elementos centrais oportunidade, conhecimento e experiência, e percebeu-se na justificativa dos alunos a grande ocorrência de elementos relacionados à aquisição de conhecimento e experiência, na busca por oportunidades. A presença de palavras semelhantes na distribuição das evocações no quadro de quatro casas e nas justificativas de ambos os termos sinaliza a existência de elementos comuns aos objetos de representação, e sugere uma possível relação entre eles. Tanto o ensino técnico quanto a capacitação profissional estão inseridos em um quadro mais amplo de referência, o da Educação Profissional, que historicamente tem sido alvo de conflitos entre formar profissionais para o mercado de trabalho e formar cidadãos para a vida em sociedade. Destaca-se a forte presença do termo oportunidade, em ambos os quadros, reflexo do que 
Bourdieu (2015) denomina de ideologia da "escola libertadora", a ideia de que a escola seria o espaço de oportunidade de crescimento e ascensão social na sociedade capitalista de classes, principalmente para os jovens dos estratos de classe menos favorecidos.

Figura 2. Quadrante das evocações ao termo capacitação profissional

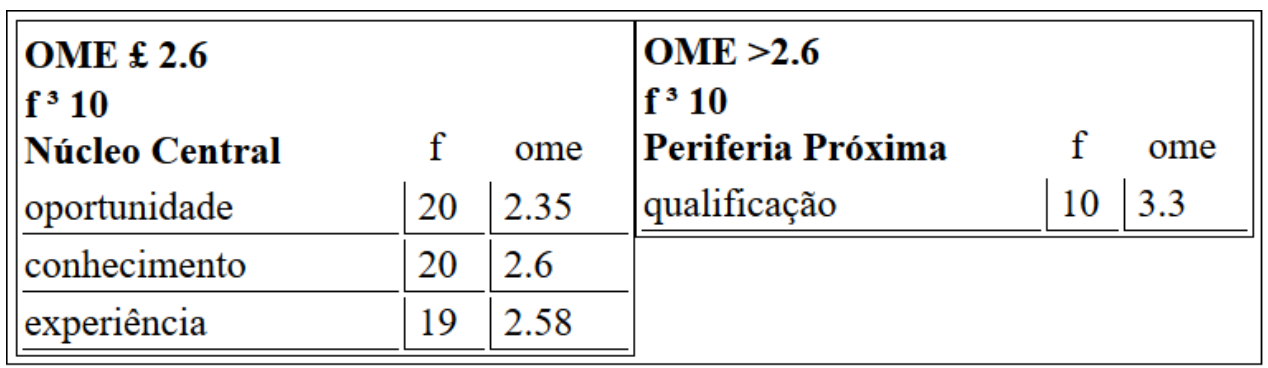

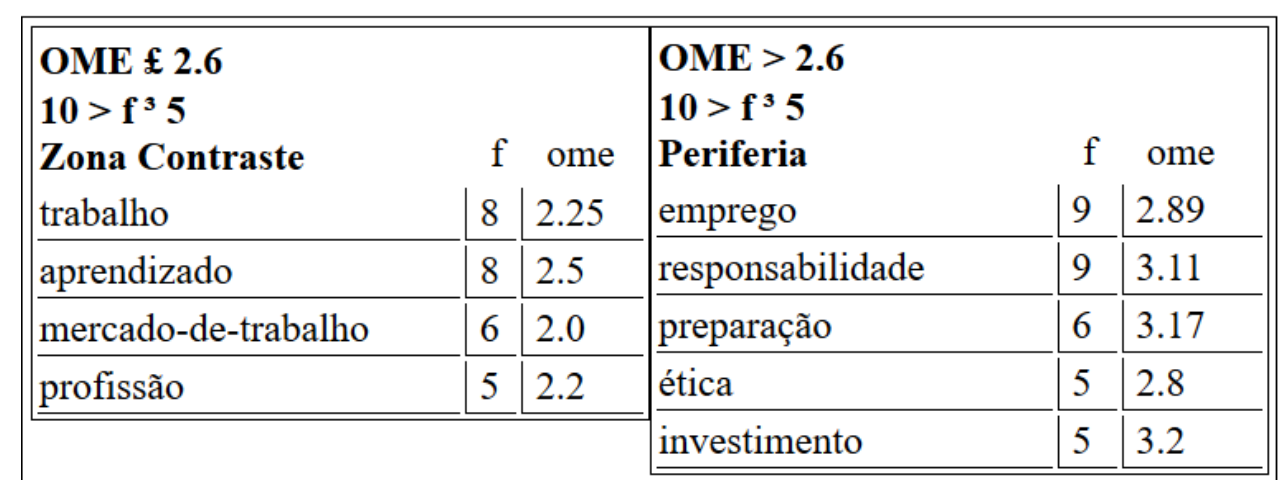

Fonte: Brandão, 2016.

As justificativas dos participantes da pesquisa para a escolha do termo oportunidade, possivelmente central em ambas as representações, demonstram o caráter polissêmico desse elemento e as contradições presentes na educação profissional, pois, embora essa modalidade de educação configure-se como uma possibilidade de preparação para o trabalho, as oportunidades são escassas e a capacitação deixou de ser garantia de ingresso no mercado de trabalho, já que não há equivalência entre formação, nível de conhecimento e os postos de trabalho disponíveis, principalmente para o jovem em busca da primeira experiência de trabalho. 


\section{OS SISTEMAS DE REPRESENTAÇÃO NA EDUCAÇÃO EM SAÚDE}

Em uma pesquisa-ação com o objetivo de investigar a relação entre as representações sociais de saúde e atividade física, bem como as condições que favorecem a adesão à prática de exercício físico (BENEVIDES, 2014), é apresentado um exemplo de como a concepção de sistemas de representações sociais pode auxiliar na intervenção em educação para a saúde e na transformação das práticas e representações.

Participaram da pesquisa 108 funcionários de uma rede de supermercados do município de Fortaleza, Ceará, 44 mulheres, com média de idade de 28 a 43 anos, e 64 homens, com média de idade de 28 a 45 anos, aos quais foi ofertada a possibilidade de realizar atividades físicas gratuitamente. Os participantes passaram por uma avaliação física antes e três meses após a intervenção realizada pela pesquisadora, como forma de mensurar os resultados das atividades desenvolvidas.

Foi utilizado um instrumento de avaliação física, construído especificamente para a pesquisa, no intuito de determinar o perfil do grupo e acompanhar a evolução dos participantes durante o estudo. O instrumento de avaliação física era composto por medidas do índice de massa corporal (IMC), percentual de gordura e circunferência da cintura, e serviu de base para a confecção de um programa de atividades físicas. Além desse instrumento, os participantes responderam ao Questionário Internacional de Atividade Física (IPAQ), para se ter uma estimativa da frequência com que praticavam exercício físico. Por meio da avaliação física inicial, verificou-se que a maioria dos participantes da pesquisa estava em uma condição de sobrepeso ou de obesidade, evidenciada pela escala do Índice de Massa Corporal (IMC).

Para a pesquisa das representações sociais de saúde e atividade física, foi utilizado um questionário de evocação livre, com a seguinte pergunta: "[...] quais as palavras ou expressões que vêm espontaneamente à sua cabeça quando você ouve a expressão: saúde e atividade física?". As respostas foram tratadas numa análise prototípica com o auxílio do software Evoc $2005^{4}$. Os participantes foram solicitados a enumerar as respostas e justificá-las.

Para avaliar a atitude dos participantes em relação às respostas, uma escala do tipo Likert foi utilizada. O questionário também continha perguntas sobre o uso do tempo livre e as justificativas para a prática ou não de atividade física. Foram constituídos dois grupos focais de 10 pessoas para a discussão das práticas desenvolvidas pelos sujeitos e avaliar os resultados das atividades durante a pesquisa. As sessões de grupo focal aconteceram concomitantemente à intervenção proposta.

\section{Saúde e a prática de atividade física}

Os resultados do questionário de evocação livre apontam como possíveis elementos centrais da representação social de Saúde os seguintes elementos: alimentação saudável, bem-estar, esporte e exercício. Alimentação saudável foi a expressão de maior ocorrência entre as evocações, sendo citada por mais da metade dos participantes. No entanto, se as palavras esporte e exercício forem consideradas como pertencentes a um único elemento (atividade física), este seria o elemento de maior evocação. Esses elementos indicam uma representação de saúde associada a hábitos considerados positivos e um estado de bem-estar, com práticas que envolvem a alimentação saudável e a atividade física. A justificativa dos participantes para a escolha dos termos reflete uma

\footnotetext{
${ }^{4}$ Ensemble de Programmes Permettant l'Analyse d'Évocations, ou Conjunto de Programas para a Análise de Evocações, software criado por Pierre Vergès.
} 
visão idealizada de saúde, como um estado de bem-estar biopsicossocial. Verificou-se entre os participantes uma atitude positiva, porém, foram apontados motivos como a falta de tempo e dinheiro, preguiça e cansaço como obstáculos à prática de atividade física.

Já a representação de Atividade Física seria constituída pelos elementos centrais saúde, musculação, bem-estar e natação. As palavras corpo e exercício também foram fortemente evocadas, apresentando frequência expressiva. Destaca-se o elemento saúde como o mais evocado pelos participantes e a presença de diversas modalidades de exercício, que podem estar relacionadas às práticas privilegiadas pelo grupo. A atividade física poderia ser considerada um objeto mais próximo da realidade dos participantes, por ter um caráter mais concreto. Dentre os motivos elencados para a prática de atividade física nas justificativas dos participantes, surge a palavra estética, ausente do núcleo central nas evocações, mas possível elemento da zona muda ${ }^{5}$ da representação, relativa ao corpo enquanto escultura e à apresentação pessoal.

Os instrumentos utilizados na pesquisa não permitem identificar o tipo de relação estabelecida entre os objetos, mas a presença de elementos comuns sugere que as representações podem ser organizadas em um sistema, no qual a atividade física, aliada a fatores como alimentação saudável, são práticas promotoras de saúde. No entanto, a adesão às práticas está condicionada a fatores objetivos e limitações individuais como tempo e dinheiro.

Durante a intervenção, a realização de grupos focais permitiu a aproximação entre os participantes e uma consequente identificação entre eles, que, embora trabalhassem no mesmo local, ainda não tinham uma relação de amizade. No processo grupal, observou-se que os participantes serviram de apoio uns aos outros para a adesão às atividades realizadas, fortalecendo a intenção de efetivação das novas práticas e diminuindo o efeito dos elementos condicionais desfavoráveis.

Ao final da intervenção, foi construído um modelo de sistema de representação (Figura 3) focado na adesão às práticas e transformação das representações sociais. De acordo com o modelo, para intervir e produzir mudanças duradouras nas práticas e nas representações é necessário entender a relação que se estabelece entre os elementos das representações que compõem o sistema cognitivo de saúde e atividade física, as condições objetivas favoráveis ou desfavoráveis à prática de exercício físico e o envolvimento afetivo dos sujeitos com o objeto de representação.

${ }^{5}$ Conceito desenvolvido por ABRIC (2003), refere-se a elementos que, devido a uma forte pressão normativa, não são abertamente expressados pelos participantes. 
Figura 3. Sistema de representação, práticas comunicativas e condicionalidade

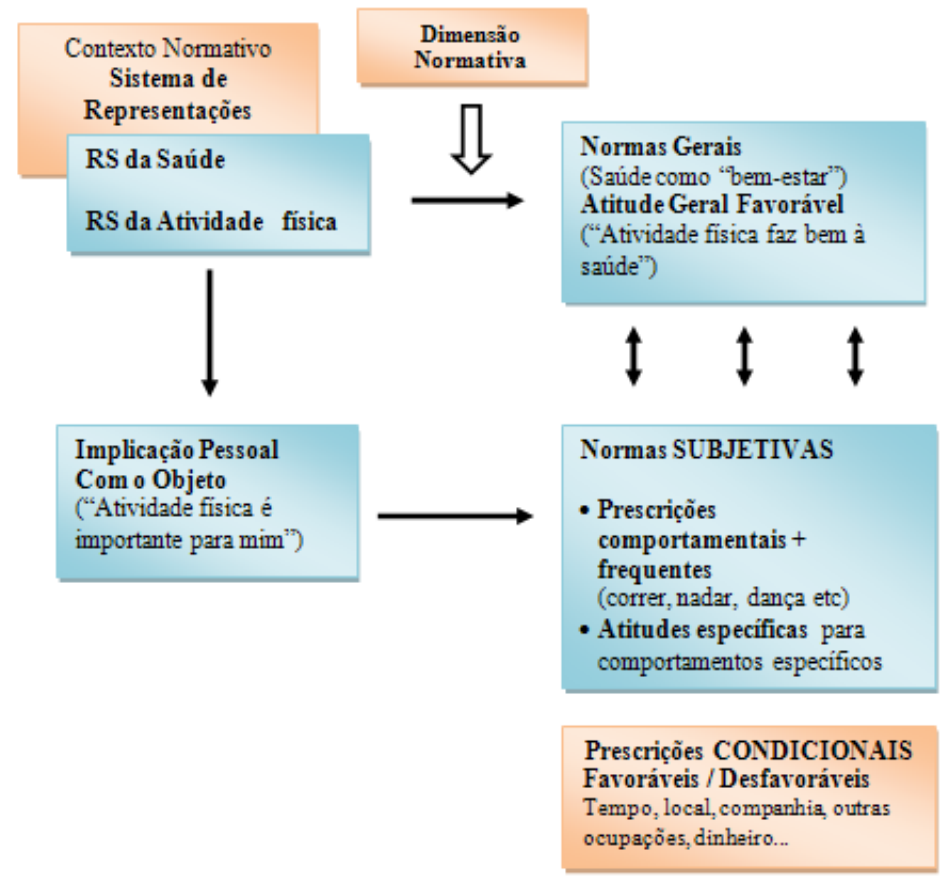

Fonte: Benevides (2014)

\section{CONSIDERAÇÕES FINAIS}

Garnier (2015) afirma que para responder aos desafios apresentados pela interdisciplinaridade e a complexidade a Teoria das Representações Sociais pode beneficiar-se de uma perspectiva sistêmica, já presente em algumas de suas vertentes ou abordagens, que permita a contextualização dos objetos e dos grupos em situações reais. A noção de sistemas de representações sociais presente nas pesquisas embasadas na Abordagem Estrutural oferece uma alternativa holística para a pesquisa em Educação, favorecendo a construção de modelos transdisciplinares para a compreensão e intervenção nas práticas educativas.

Um sistema de representações pressupõe a organização dos objetos de representação social em conjuntos ou redes, nas quais novas representações tendem a ser inseridas, de forma a adquirir significação e estruturação. Devido a essa organização em rede, mudanças no ambiente e nas práticas referentes a um objeto social podem ocasionar a transformação de uma ou mais representações pertencentes ao sistema, numa espécie de transformação relacional (PIANELLI, SAAD, 2016).

A noção de sistemas de representações sociais oferece possibilidades para o aprofundamento do olhar psicossocial sobre as práticas no campo da Educação, condição necessária para a promoção da mudança social (ABDALLA, VILLAS BÔAS, 2018). Nas duas pesquisas apresentadas, foram apontados indícios da relação entre objetos de representação social, no entanto, ainda é necessário desenvolver metodologias que permitam a investigação e intervenção em práticas sociais e representações, considerando a relação entre os grupos no contexto educativo. 


\section{REFERÊNCIAS}

ABDALLA, Maria de Fátima Barbosa; VILLAS BÔAS, Lúcia Pintor. Um olhar psicossocial para a educação. Cadernos de Pesquisa, v. 48, n. 167, p. 14-41, jan./mar. 2018.

ABRIC, Jean-Claude. Pratiques sociales et représentations. Paris: Presses Universitaires de France, 1994. ABRIC, Jean-Claude; CAMPOS, Pedro Humberto Faria. Les éducateurs et leur représentation sociale de l'enfant de rue au Brésil. In: ABRIC, Jean-Claude (org.) Exclusion sociale, insertion et prévention. Saint-Agne: Erès, 1996. p. 137-149.

ABRIC, Jean-Claude. Abordagem estrutural das representações sociais: desenvolvimentos recentes. In: CAMPOS, Pedro Humberto Faria; LOUREIRO, Marcos Corrêa da Silva (orgs.). Representações sociais e práticas educativas. Goiânia, 2003. p. 37-57.

BENEVIDES, Andréa Cristina da Silva. Sistema Sócio Cognitivo e adesão ao exercício físico: práticas comunicativas e mudança nas representações sociais no contexto da educação para a saúde. 223p. Tese. Doutorado em Educação, Universidade Estácio de Sá, Rio de Janeiro, 2014.

BOURDIEU, Pierre. Os excluídos do interior. In: BOURDIEU, Pierre. Escritos de Educação. NOGUEIRA, Maria Alice; CATANI, Afrânio (orgs.). 16. ed. Petrópolis: Vozes, 2015. p. 243-256.

BRANDÃO, Caio Teixeira. Ensino Técnico e Capacitação Profissional: um relato de pesquisa no Instituto Federal do Amapá. In: NEGREIROS, Fauston; SOUZA, Marilene Proença Rebello (orgs.). Práticas em psicologia escolar: do ensino técnico ao superior. Teresina: EdUFPI, 2017. p. 212231.

BRASIL. Ministério da Educação. Lei n. 11.892. Brasília, DF, 2008.

BREAKWELL, Glynis M. Social Representations and Social Identity. Papers on Social Representations, v. 2, n. 3, p. 1-20, 1993.

BONA, Viviane; ESPÍNDOLA, Elisângela Bastos de Melo; MAIA, Lícia de Souza Leão. As representações sociais de autonomia e autonomia docente e suas relações de encaixe. Revista Educaşão e Cultura Contemporânea, v. 14, n. 37, p. 161-183, out./dez. 2017.

CAMARGO, Brígido Vizeu; WACHELKE, João. The Study of Social Representations Systems: Relationships Involving Representations of Aging, AIDS and the Body. Papers on Social Representations, v. 19, p. 1-21, 2010.

CAMPOS, Pedro Humberto Faria. Rapports entre pratiques et représentations: apports théoriques et empiriques pour un modèle d'étude. Psychologie \& Société, v. 3, n. 6, p. 135-166, 2003.

CODOL, Jean-Paul. On the system of representations in a group situation. European Journal of Social psychology, n. 4, p. 343-365, 1974.

FÉLIX, Lívia Botelho; ANDRADE, Danyelle Almeida; RIBEIRO, Fernanda Siqueira; CORREIA, Clarissa Cristina Gonçalves; SANTOS, Maria de Fátima de Souza. O conceito de Sistemas de Representações Sociais na produção nacional e internacional: uma pesquisa bibliográfica. Psicologia e Saber Social, v. 5, n. 2, p. 198-217, 2016.

FLAMENT, Claude. Aspects périphériques des représentations sociales. In: GUIMELLI, Christian (Éd.). Structures et transformations des représentations sociales. Lausanne: Delachaux et Niestlé, 1994, p. 85-118.

FLAMENT, Claude; ROUQUETTE, Michel-Louis. Anatomie des idées ordinaires: comment étudier les représentations sociales. Paris: Armand Colin, 2003. 
GARNIER, Catherine. La genèse des représentations sociales dans une perspective développementale. In: ROUQUETTE, Michel-Louis; GARNIER, Catherine (orgs.). La genèse des représentations sociales. Montréal: Nouvelles, 1999. p. 87-113.

GARNIER, Catherine. Les représentations sociales: entre l'individualisme et l'holisme. TrajEthos, v. 4, n. 1, p. 9-24, 2015.

GILLY, Michel. Maître-élève: rôles institutionnels et représentations. Paris: P.U.F., 1980. 300p.

JODELET, Denise. Représentations sociales: phénomènes, concept et théorie. In: MOSCOVICI, Serge. Psychologie Sociale. Paris: PUF, 1984, p. 357-378.

MOSCOVICI, Serge. A psicanálise, sua imagem e seu público. Petrópolis: Vozes, 2012.

PEREZ, Judith dos Santos. Representaçôes Sociais de trabalho docente por professores atuantes nos anos iniciais do Ensino Fundamental: a relação entre o papel e as práticas. 155p. Tese. Doutorado em Educação, Universidade Estácio de Sá, Rio de Janeiro, 2017.

PIANELLI, Carine; ABRIC, Jean-Claude; SAAD, Farida. Rôle des représentations sociales préexistantes dans les processus d'ancrage et de structuration d'une nouvelle représentation. Les Cahiers Internationaux de Psychologie Sociale, n. 86, p. 241-274, 2010.

PIANELLI, Carine; SAAD, Farida. Environmental Changes and Dynamics of a Network of Social Representations. Papers on Social Representations, v. 26, n. 2, p. 1-7, 2016.

RATEAU, Patrick. Le noyau central des représentations sociales comme système hiérarchisé: Une étude sur la représentation du groupe. Les Cabiers Internationaux de Psychologie Sociale, n. 26, p. 241274, 1995.

RATEAU, Patrick; LO MONACO, Grégory. L'Approche Structurale. In: LO MONACO, Grégory; DELOUVÉE, Sylvain; RATEAU, Patrick (orgs.). Les représentations sociales: Théories, méthodes et applications. Ouvertures Psychologiques. Louvain-la-Neuve: De Boeck, 2016. p. 131134.

SILVA, Priscilla de Oliveira Martins; TRINDADE, Zeidi Araújo; SILVA JUNIOR, Annor. As representações sociais de conjugalidade entre casais recasados. Estudos de Psicologia (Natal), v. 17, n. 3, p. 435-443, 2012.

SOUSA, Clarilza Prado. Estudos de representações sociais em educação. Psicologia da Educação (São Paulo), v. 1, p. 285-323, 2003.

SOUSA, Clarilza Prado; NOVAES, Adelina de Oliveira; VILLAS BÔAS, Lúcia Pintor. Representações sociais e educação: panorama dos estudos desenvolvidos pela Cátedra Unesco sobre profissionalização docente. Educação e Linguagem, v. 15, n. 25, p. 19-39, jan./jun. 2012.

SOUSA, Clarilza Prado; MARCONDES, Anamérica Prado; JARDIM, Anna Carolina Salgado; COÊLHO, Vanusa dos Reis (orgs.) Qual futuro? Representações sociais de professores, jovens, crianças. Campinas: Pontes Editores, 2018.

WACHELKE, João. Les systèmes de représentations sociales. In: LO MONACO, Grégory; DELOUVÉE, Sylvain; RATEAU, Patrick (orgs.). Les représentations sociales: Théories, méthodes et applications. Ouvertures Psychologiques. Louvain-la-Neuve: De Boeck, 2016. p. 131-134.

WACHELKE, João; CONTARELLO, Alberta. Italian Students' Social Representations on Aging: An Exploratory Study of a Representational System. Psicologia: Reflexão e Crítica, v. 24, n. 3, p. 551 560, 2011. 


\section{Informações da(os) autora(es)}

Caio Teixeira Brandão

Instituto Federal do Amapá (IFAP)/ Universidade Estácio de Sá (UNESA)

E-mail: caio.brandao@ifap.edu.br

ORCID: https://orcid.org/0000-0002-0196-3074

Link Lattes: http://lattes.cnpq.br/ 6823296450872020

Andréa Silva Benevides

Universidade Federal do Ceará (UFCE)/ Centro Universitário Estácio do Ceará

E-mail: andreasbenevides@terra.com.br

ORCID: https://orcid.org/0000-0002-8202-9450

Link Lattes: http://lattes.cnpq.br/4165413685802953

Pedro Humberto Faria Campos

Universidade Estácio de Sá (UNESA)

E-mail: pedrohumbertosbp@terra.com.br

ORCID: https://orcid.org/0000-0002-0844-8358

Link Lattes: http://lattes.cnpq.br/4165413685802953 